\title{
Driving, Dashboards and Dromology: Analysing 1980s Videogames Using Paul Virilio's Theory of Speed
}

\author{
Alex Wade
}

\section{Speed and Inertia}

Space and time is of central concern of Paul Virilio. Just as Lefebvre and Merleau-Ponty were concerned with the reductive effects of the scientifically conceived spaces of technology upon everyday life, so Virilio extends these concerns to the impact of time upon lived, or 'real space.' As techniques and technologies of managing time are applied to everyday life, so 'real spaces' are progressively diluted and individuals can no longer delineate between the private and the public: so called 'dead time' on mass transit systems is elided by Wi-Fi connectivity; Internet connected appliances are always-on for corporations to target advertising and governments to harvest data to increase national security, with the PRISM program showing little demarcation between either sphere. Inhabitants of contemporary societies are overlaid with a gossamer digital net, allowing spaces to be traversed in advance: GPS positioning covers the globe, permitting navigation and simulation of spaces pre-arrival, Google's variant navigation software shows topographical and actual views of journeys before they take place, war games provide tactical and strategic supremacy of potential theatres of battle, flight and medical simulators allow a view of future egocentric (airspace) and exocentric (the human body) spaces before any actual flight or operation is undertaken. Laborsaving devices privilege real-time over real-space, the result being that the temporal obliterates the spatial, "here no longer exists, everything is now' (Virilio 2000b: 125).

The increasing reliance on machines reveals an urgent message which is central to Virilio's exposition on time and space. For instance, in the highly conceived, digital space of modern aeronautics, the pilot, crew and passengers are ensconced within the 'speed machine' of a metallic capsule, supplemented by electronic prostheses, which transforms the measurement of distance (space) into the measurement of duration (time). Thus, when passengers board a Boeing 747 bound for Los Angeles, they are instructed as to how long rather than how far they will fly, so that space is progressively reduced to its lowest common denominator, and the journey elided behind departure and arrival. The folding of space into time is achieved through the very speed of air travel: as duration decreases, so the room available to the passengers decreases, creating an inability to physically move around the cabin, a condition enabled by the personal consoles of music, films and games, ultimately generating a somnambulant desire for inertia. As speed increases, so space becomes reduced to a point, 'no doubt there will be no longer anything but arrival, the point of arrival, the departure will itself have disappeared in the instantaneity of the projection' (Virilio 2008: 110). The 'real spaces' of perception and lived space are summarily reduced, as inertia becomes a way of life. This is apparent in the everyday too, where individuals who are inert as they cannot summarily move through space under their own motility are provided with the prostheses of electric wheelchairs. Here they become part of the trajectory of the machine. The inert are the disabled fitted out with prostheses to realize a prototype for future human/ machine integration 'the able but overequipped air force pilot resembles in every feature the equipped invalid' (Virilio 2000a: 65). The collapse of space into time is fully realized in digital space, placing us in the same position of inertia as the invalid or the aeronaut, evident in the mundane, but telling examples of shopping for books from Amazon, 
or downloading videogames via Steam. For the armchair traveler commodities, communication and services are delivered on demand, with the maximum of commutable speed and the minimum of physical movement.

\section{The Effects of Speed}

With the technologies of the digital, the amount of 'space' available to the user is of unequivocal importance. The quantity of information which can be stored on a magnetic media has moved from bits, to bytes; kilobytes to megabytes; gigabytes to terabytes; the performance of a graphics card is measured in the availability of RAM, with the rider that miniaturization diminishes the physical presence of this in lived space by continually reducing the size of the desktop PC, tablet, smartphone or digital camera, with the current trend being towards wearable technologies, e.g. smartwatches or eyewear. As digital space increases in prevalence and importance by both enmeshing, overlaying and supplanting other spaces, there is a requirement to navigate with greater ease and rapidity. Therefore, as the storage space available to digital technologies increases, so does the speed of navigation, with the astronomy of numbers applied to tech representative of one thing: speed. CPUs are overclocked to wring the final iota of performance from searing silicon; mathematical calculations are measured in teraflops; communication speed is constituted by gross bit rate.

With the reduction of time taken to process a calculation concurrent with the increase in space, speed allows traversal between the co-dependents of space and time. With the current archetype of digital space being the Internet and its emphasis on the maximal communication of information in the shortest possible time, the reality of information is entirely contained in the speed of its dissemination. This theorem partly explains the exponential rise in the popularity of personal communication hardware and the social networks linked into them. Here, it is not the content which is important, but the medium, speed, which ensures that information is only of any value if it is delivered fast; better still that speed is information itself!' (Virilio 1995: 140). Therefore the medium is not only the message, but intrinsic to lived experience, evidenced in the incessant use of social media, keyboard and keypad, which via speed of response, allows navigation of a proliferating space with increasing brevity.

In this digital space, it is the user who is projected and distanced towards/from the subject or object. However, as I show below in the specific examples of the impact of speed upon the videogame, the time manifest in digital space simultaneously brings objects and surroundings towards the user, with dromology becoming, paradoxically 'the wait for the coming of what abides' (Virilio 2008: 110) as everything arrives without the need to depart. So, while the space of the digital projects the user towards the subject/object, the time of the digital projects the subject/ object towards the user: speed acts as the medium between the two. This simultaneous and complementary fusion of space and time assumes control over ego-centric (introverted) space, in lieu of exo-centric (extroverted) space. The proclivity is towards travelling without moving: as users navigate with GPS and Google Earth, so they are locked into an inertia modulated by access to a screen via interface. Thus, technocratic societies with their reduction of interaction within tangible, physical spaces are seeing a 'progressive disappearance of anthropological-geographic reference' (Virilio 2000a: 68). Potentially, the employment of speed as a medium of transmission in the spatial and temporal realms has catastrophic consequences for 'real spaces' as instantaneity and ubiquity will abolish space along with the interval.

For the user of digital space, everything can be satisfied in the present chronos. Travelling the world can be reduced to instantaneous navigation and flattened onto the 'square horizon of the screen' where there is 'no more delay, no more relief' (Virilio 1997: 26). The archetype for this space was once the negative horizon of the desert, where the attempts of land-speed records rendered objects and landscapes a homogenous and dromogenous hell of the same (Virilio 2008: 134), but in the new model the digital stretches around the globe like cling-film, which falsifies the depth, the length, the distances of perception of time and space. Just as the motor car or civil aviation bring faraway lands closer to us, so in the digital the medium of speed distances us from the tangible subject or object. Such is the promise of digital space: to project and distance oneself, to instantly communicate, to homogenize and smooth out all spaces, so that all users are locked into a universal time of instantaneousness, without having to wrest physical bodies from inertia.

Yet the holy grail of many audiovisual technologies - and especially videogames - is to provide the illusion of topographical relief. This is evident in early era games, where a top-down, God's eye view generates a distance between player and avatar, before becoming more pronounced with the increase in processing speed. In order to optimize the illusion of relief, early commercial videogames employed the artistic technique of parallax scrolling, 
where landscapes on the screen are refreshed at different rates, so that the ground in front of the player would move quicker than the mountains in the background. Such was the success of the technique that it lent itself to the title of Sensible Software's sci-fi shooter Parallax (1982). At the same time, developers experimented with isometrics to create the illusion of '2.5D' games, such as Zaxxon (1982) where the player would view the landscape from the corner of the screen. The effect was to provide the illusion of 'flying into' the screen, which, in a considerably updated format, provides the basis for the 3D representations of space seen in today's polygon-rendered first person shooters. With this rejoinder in mind, the second part of this paper will examine how Virilio's work can be used to analyze videogames of the 1980s where a variety of techniques, tricks and technologies were employed to convey ideas of speed and substitution in the videogame.

\section{Virilio and the Videogame}

The increase of speed and its correlated rise in inertia is a feature integral to technocratic societies. With each increase in speed, bodily movement is reduced to a perfunctory action. Travellators at airports allow people to move around with the minimum effort, preparing the traveler for the zero degree inertia of trans-Atlantic flight. Autobahns permit unlimited automotive speed, with the driver ensconced in a cabin bristling with technologies, rendering the driver obsolescent (Virilio 2008: 107). This has reached its logical conclusion with the use of the 'Reaper', an Unmanned Aerial Vehicle (UAV) in Afghanistan, which can stay airborne for longer than conventional aircraft as the inconvenience of the human pilot is removed. There is no need for a cockpit, life support systems or radio as the aircraft is controlled from Creech Air Force Base in Nevada, USA. A BBC report on the UAV stresses that control of the drone is 'no videogame' and yet the image of the RAF Wing Commander, chatting to the reporter while sitting at a console replete with screens and an Xbox 360 joypad as the Reaper flies on a non-stop 20 hours reconnaissance mission 7000 miles away from the gamer/pilot is clearly the manifestation of pure war, which transcends its normative area of conflict. While technologies of projection and distancing obsolesce the individual pilot, a gamer is generated in their place who, as outlined above, experiences a stretched cling-film of depth-free space. It is no surprise then, that the shimmering flatness of the screen is reflected in the mirage (1) of desert wars of the 21st century, and subsequently doubled in the desert setting of the UAV's control base in Nevada, a location so acquiescent to technologies of speed that vehicles from the last three world land speed records have been tested, developed, deployed and set there, in each case further increasing the inertia of the pilot of these vehicles powered by aerospace engines. The dromological effects of speed and inertia are so manifest in the experience of bodies who accelerate through space that

He who gets behind the wheel of a racing car ... completes his natural stereoscopic vision with a new type of prosthesis of vision capable of providing him with the mobile illusion of a kinetic transformation of his field of vision, the optical illusion being perhaps here only that of an alleged relief of perspectival space

(Virilio 2008: 133)

In a similar way, dromology, Paul Virilo's term for the study of speed, is relevant to the significance of driving/ racing games, which throughout the course of the history of videogames have proven to be one of the most popular and enduring genres. From the very first racing videogame Gran Trak 10 (1974), complete with three-spoke steering wheel, through to the 'car porn' of the Gran Turismo (1997-) series, the driving game has been at the forefront of innovation in design, high audiovisual standards and the use of prostheses or peripherals to project and maintain the illusion of speed. Indeed, it is through the driving genre that the importance of speed to the study of the videogame becomes apparent, as the use of the 'vehicle' in the videogame is not merely limited to the driving genre. To outline this I will discuss a variety of videogames, chiefly from the 1980s where the use of speed is crucial to their impact and success, and the parallels they have with Virilio's theory of speed.

\section{| Dromology and the Game of Driving: Monaco GP and Motor Mania}

The use of the screen as a device to fuse inertia and speed belies a striking similarity between the videogame 
and the vehicle, which requires that the users or occupants assume a trans-spatial approach 'the framed opening of the windshield is not therefore a window but rather a window-door which the passengers pass through without stopping' (Virilio 2008: 104). The effect of speed, where time and space collapse and extend into the vanishing point is enchanting for the auto-dweller, as the (wind) screen, acting like a looking glass, entices and permits us to view the future that we are hurtling towards. In the vehicle, passengers are inert, as the surroundings move dromoscopically around them, a technique employed by racing games to project the illusion of speed. While early racing games were represented on one screen and didn't alter throughout the duration of the game, as with Sprint 2 (1974), with the increase in processing speeds the practice of projecting the landscape onto the player's on-screen vehicle became the norm. This is especially evident from the 1980s onwards and can be seen in Sega's Monaco GP (1980), where corners would rush down the screen towards the player's car and in Motor Mania (1982).

Monaco GP was one of the first arcade games to feature a 'deluxe' model, where the player could sit down as if embedded in the cockpit of Formula 1 car, causing the player to be more inert than if they were standing at the upright model. Furthermore, although the orientation of the player in Monaco GP was horizontal, the monitor was vertical, as opposed to the horizontal orientation of later games, where the emphasis tends towards width rather than length. This may appear to be a trivial observation, but it is important to note that early driving games, in spite of moving the landscape towards the player, did not attain pure speed. The vertical orientation of the monitor is akin to an exploratory desire as seen in exo-centric space and exo-colonisation. The definitive example of this orientation is the ballistic positioning of astronauts in rocket propelled vehicles: vertical/ballistic orientation is literally and metaphorically a wish to reach for the stars. When the monitor moves towards the horizontal and there is a letterboxing of vision there is a tendency towards insularity, ego-centric space and endo-colonisation. This is best seen in the movement away from the exploration of the cosmos to the exploration of genetic code, where humans orbit human bodies, rather than celestial bodies. The movement to horizontal orientation is widespread and particularly apparent in screen-based technology, from the letterboxing effect of the now-ubiquitous 16:9 screen ratio to the advent of 3D representations in videogames, cinema and television broadcasts (Schroter, 2014). The outcome is that objects in the center of the screen of the gamer/driver scroll quicker than those on the periphery. The image is convexed and depth is generated to the field of vision. Known as 'motion blur', this visual technique is used extensively in contemporary racing games, with Sony Liverpool's WipEout HD (2008) augmenting the mirage further by supporting the use of 3D glasses. Contrary to Virilio then, the irony becomes apparent: the faster the vehicle the greater the relief.

If Monaco GP generates the illusion of speed, then Motor Mania produces a representation of speed. As the landscape scrolls down the left hand side of the screen towards the player's car, the gamer/driver is required to observe and assimilate additional information from the 'dashboard' on the right hand side of the screen. The dashboard takes the form of a plethora of gauges including a speedometer, milometer, fuel gauge and voltmeter. As the dashboard monitors and informs the position of the gamer/driver through the landscape, it also narrows the space of the (wind)screen, 'shrinking its passengers field of vision, the frame of the dashboard gives rise to an acceleration of the sequencing that reinforces the effect of the acceleration of the vehicle' (Virilio 2008: 103). Although the driver can see the landscape, the dashboard instruments permit a clearer view of the outside than the windscreen alone: a stereoscopy which serves as a double reduction to the distance-time of the trip and the letterbox framing of the (wind) screen. Horizontal orientation is discernible in Motor Mania where the dashboard takes up $50 \%$ of the screen, with the projection of information onto the gamer/driver of equal importance to the representation of the landscape.

\section{Battlezone: Territories Unknown}

As the above example of the Reaper UAV shows, the supplanting of human input by technology is a way of minimizing risk to those engaged in the execution of pure war. The ultimate desire of combat is to be absorbed into a digital space, to 'stay out of reach, all the while remaining present' (Virilio 1986: 39). This is an aesthetics of disappearance, of camouflage, where the warrior, via technology, removes the body from the war-zone. Disappearance is of tacit - and tactical - importance to the mechanized divisions of land armies, where tanks project false appearances and tank commanders, gunners and drivers are ensconced in a composite ceramic shell, shielded from the elements of war and nature. 
There is the intimation of disappearance in Atari's Combat (1977), where, in one play mode, the tank is invisible to the players except for a few seconds after a shot is fired. However, it is Battlezone (1980), where the game, the tank, the gamer/driver and the dashboard are interfaced, seamlessly merged and identified 'with a victorious vision, to the point where the dashboard comes to seem rather like a misunderstood game of war' (Virilio 2008: 107). The player of Battlezone controls a tank in the first person perspective, but disappears as the gamer and the screen are hyper-mediated through the use of peripherals. The vanishing of the gamer/driver is shown in three separate but interconnected ways. First, the player views the landscape through a set of goggles, similar in form and usage to a periscope on a tank or submarine (and perhaps anticipatory of Google Glass technology). To move the tank, the player must manipulate two joysticks, representing the caterpillar tractors which propel the tank. Second, through the use of a radar screen at the top of the display, the game substitutes the warrior for technology, as the Doppler optics serve to augment the eyesight of the tank commander by bringing the vast expanses of the battlefield into close proximity, convexing the space of the battlefield through the periscope and radar. Third, the innovative 'vector' graphics used in Battlezone are particularly instructive as to the role of the tank in modern warfare. The use of a three dimensional representation of a landscape counters the flattening of space by opening the battlefield to depth, so 'the technology of vectors thus comes to replace the tactics of bodies' (Virilio 2008: 107). The use of vectoring means that the gamer/warrior can predict with some certainty the movements of the enemy in space, and therefore anticipate what will happen in the time of the future. Similar to the gamer/driver of Monaco GP with 'the prevision of the movement of the opposing horizon for the driver similar to the prevision of the enemy for war leaders' (Virilio 2008: 107), it is possible to see Battlezone as part of a wider project of a rigorous management of time and coincident social impact. First, Battlezone was adapted for use by the US Army as a trainer for their M2 Bradley Fighting Vehicle. Bradley Trainer (aka Military Battlezone) was considerably more complex than its arcade variant as it incorporated gravitational effects which altered the trajectory of the shells and identifiable targets, so trainee tank commanders wouldn't friendly fire their allies. As one of the first military uses of videogames, Bradley Trainer identifies with what Virilio describes as a 'sophisticated form of Kriegspiel [war game] . . dromoscopy would be in some ways a video game of speed, a Blitzkriegspiel [Lightening war game]' (Virilio 2008: 107). Therefore, in Battlezone speed, technology and gaming anticipates Der Derian's (2001) Military Industrial Media Entertainment Network (MIMENET) and Stahl's 'militainment' (2010) which, in fast, but highly controlled societies, are concepts vertically integrated into videogame technologies. Second, as with the notion of the gamer/driver seen in Motor Mania and Monaco GP, it is Battlezone's landscape which moves around the player, but, unlike other games of its era, it is the player who controls this movement. Battlezone's desire for speed is a witness to the simultaneous desire for control over that speed, as the more mobility increases, the greater the desire for control. As shown at the end of this article, the development of the dynamic of the control of the speed found in videogames is crucial to Virilio's analysis of wielding control over entire societies.

\section{The Inertia of Speed: Pole Position II}

Battlezone's move to a three-dimensional, first-person representation of a landscape was indicative of a widespread shift towards the dashboard viewpoint of the gamer/driver. This graphical representation, where the player is situated elevated and behind the car, or as a fly on the windscreen are termed 'third-person' or 'first-person' perspectives respectively. Pole Position II (Namco/Atari, 1983) with its inclusion of the Monaco GP deluxe model of arcade cabinet used the third person perspective while the gamer/driver races around a variety of Japanese Formula 1 courses. The difference between Pole Position II and the earlier first-person perspective of Battlezone is the use of sprite-based graphics, which enables the use of a wider variety of styles and colors. However, the use of sprite graphics has unforeseen consequences, for when the player reaches top-speed, the movement of the track towards the player is so quick that that it appears not to move and then, eventually, appears to move backwards. This is the mastery of 'real speed', so desired by F1 drivers, where to approach obstacles with such velocity is to have 'the impression that you are moving in slow motion' (Prost cited Virilio 2000a: 15) so that distance-time is abolished between objects on the landscape and the gamer/driver. Pole Position II's 'wagon-wheel' aliasing - the technical term given to an image that appears to stand still when it is drawn - is the essence of passivity, where spasmodic speed causes inertia. This is manifested twice: first embodied in the player who sits prone in front of the screen, then supplemented by the suspended animation of the stationary image, so that car speed and audiovisual speed 
are rendered compatible. The twin poles of inertia evident here are experienced by the gamer and driver. Both are subject to the sensation of projection and distancing across time and space, but the videogame is doubly digital as it is a hyper-realization of an already digital experience,

Dromovision, (automobile media) simulates the fleeting well before television (audiovisual media) simulates proximity . . . up to the moment hardly imaginable where instantaneity and ubiquity will abolish space along with the interval, making the dromovisual apparatus the perfect equivalent of the audiovisual in a single stroke!

(Virilio 2008: 51)

The effect of the inert image on the perception of space and time by the gamer/driver betrays the ultimate aspiration of the automobile to mimic the image, or even to be superseded by it, as the image is the only high performance vehicle, the real time image which is supplanting the space where the car still moves from one place to another' (Virilio, 2000a: 14). This deftly explains why the driving genre has extensive videogame lineage and longevity as it is the digital's fundamental aim, from its genealogy in the vehicle of speed, to intensify everything into a vanishing point. The suspended animation of Pole Position II is a digital prototype, with (in)action reduced to the click of a micro-switch rendering the user distant and inert, the gamer/driver becoming an alias to the objects passing by so fast we can no longer see them move.

\section{| Vanishing Point: TX-1 and OutRun}

The example of Pole Position II is not unusual among arcade games of the early 1980s but as it is the illusion of speed, rather than inertia, which is the desirous experience of the gamer/driver, ever-increasing clock-speeds of CPUs subsequently allowed hardware to portray speed with greater sophistication in sprite-based games. Namco's TX-1 (1983) was one of the first games to properly achieve this by delineating landscape features through the use of bright, bold colors to effectively contrast between the sky and the land. These graphics are complemented by the representation of the road employing the then widespread artistic technique of placing alternate horizontal black/light grey lines on the track to help generate the illusion of speed. Nevertheless, TX-1's key innovation was the inclusion of three monitors in a deluxe cabinet. These act as peripherals in two ways: first in that they produce a peripheral vision for the gamer/driver while they concentrate on the disappearing horizon of the main monitor. Second, they allow the gamer/driver to view more of the course than in games such as Pole Position II as it is possible to see 'around' corners by glancing directly into one of the horizontally orientated monitors to the right or left of the main screen. This technique mimics the windscreen and side windows of a conventional automobile, 'with the rear window, with its windowed doors and its front windscreen, the automobile forms a quadtriptych where the travel lover is the target of a permanent assault that recalls the perspective of the painting' (Virilio 2008: 106). As outlined above, at this juncture of the development of the driving game, perspective through artistic manipulation is vital to the perception of speed, and the quadtriptych of the three screens allows for the simulation of the passenger compartment. This is a crucial step forward for the videogame as substitute for the automobile, as in the driver's seat the immediate proximity matters little, the only important thing is what happens at a distance, so as the speed of the vehicle, processor and image multiplies, so distancing in space increases 'the greater the speed, the more distant the horizon' (Virilio 2008: 106). TX-1 contrived to distance the horizon by offering the gamer/driver a choice at each checkpoint which, combined with the epigram 'Time Extend!' shows how the future projection of the trajectory encourages acceleration through space. The inclusion of multiple screens which distance the horizon in TX-1 via the use of artistic techniques, peripherals and peripheral vision, is significant in that it realizes the desire for horizontal escape, enshrined in the history of Quattrocento perspectives where the high and low (i.e. the vertical or exo-centric space) are sequestered in favor of the vanishing point.

The introduction of Sega's OutRun (1986) to arcades was a seminal cultural event in the history of videogames. OutRun redefined the driving genre by replacing the sterile racetracks of Monaco GP and TX-1 with dazzling landscapes from the surf-lapped highways of California, the big skies of Arizona to the sun-dappled byways of Martha's Vineyard. In actuality, although OutRun is rightly remembered as the pinnacle of sprite based racing games, it is best described as a game which coherently integrated all of the best features of preceding titles into one videogame. The bright red deluxe cabinet with brake, accelerator and two-speed gearbox is modelled on the iconic 
Ferrari Testarossa and, as was Sega's wont at the time, used hydraulics to simulate the movement of the vehicle. Sega's emulation of fairground rides - a first in videogames - where the individual is prostrate while propelled across two axes meant that crowds would gather to watch as players traversed American blacktop. Opulent in its presentation, OutRun is nostalgically commemorated as allowing the gamer/driver to choose from three distinctive music tracks, displayed on a radio set into a plush dashboard, the art of the motor flaunting the technique and the technology to the to the throngs of watching voyeurs-voyages. In addition to the sophistry of the dashboard, OutRun's core gameplay dynamic is the use of a 'goal' system which, akin to TX-1, requires the player to make a series of choices to reach the end of the game. In order to make these choices and reach the goal, the gamer/driver must arrive at each checkpoint before the countdown reaches zero, in order to trigger 'time extension'. Indeed, the very title of the game, 'OutRun' betrays the basest desire of gaming - and driving - what is it that the gamer/driver is trying to outrun?

\begin{abstract}
With the speed of the continuum it is the goal of the voyage that destroys the road, it is the target of the projectile-projector (of the automobile) that seems to trigger the ruin of the interval, it is the fleeting desire to go right to the end as fast as possible that produces in the opening out of the travelling, the tearing apart of the landscape.
\end{abstract}

(Virilio 2008: 105)

The concentration of the gamer/driver on the countdown, so that zero-hour is never encountered shows how space is shredded by time, evidenced in the measuring of a trip by train or airliner by duration rather than distance. When the goal supplants the journey and the ends elides the means, 'the world becomes a video game, a game of transparency and transpiercing' (Virilio 2008: 103). OutRun's dedicated and eponymous hardware meant that it could incorporate radical artistic innovations to simulate the transpiercing of the landscape. This was achieved by making the perspective of the game inert in relation to the car, so when the car moved through the landscape the perspective remained fixed, as if a camera was affixed to the rear of the vehicle. This intensifies the inertia of the gamer/driver, so that while the camera remains stationary behind the Ferrari, the vanishing point also remains constant and infinite. The outrun across broad savannahs and narrow canyons, so magnificently realised in OutRun, becomes an outrun to the strategy of the beyond, a cyclopean focus on an instant, intangible vanishing point, a space that distances while projecting.

\title{
| Concentration and Control of Speed: Operation Wolf
}

OutRun heralded an extended period of innovation in arcade manufacturing, with powerful hardware and bespoke cabinets becoming the norm. Taito's Operation Wolf (1987) invited the gamer to assume the role of a Special Forces operative on a plausibly deniable mission in an unnamed south East Asian country. While shooters were especially popular in the 1980s, they generally presented an abstract side-on, perspective, with the sidereal R-Type (1987) the paragon of the shmup genre. Operation Wolf, however, situates the player in a first person perspective, not dissimilar to that found in the classic Duck Hunt (1984). However, instead of being static, the screen scrolls horizontally, locating the warrior on an armored vehicle, or on the last level, a turbo-prop airplane. This illusion is reinforced through the utilization of peripherals in the game, with the horizontal/linear formation of the gamer/warrior 'being the means of maximal exploitation of firepower' (Virilio 2008: 58). To maximize the exploitation of firepower, the cabinet is equipped with a scale-model Uzi machine-pistol which utilizes haptic force feedback to replicate the firing of a gun. In addition the screen displays a dashboard, which tots up the logistical status of the battlefield by showing the amount of magazines, grenades and health remaining; hostages saved and, most pertinently, the amount of enemies killed/alive. This information is presented to the player, without their needing to identify the enemy's position in battle, so 'the art of the control panel, therefore appears on the one hand like a substitute for the hunt and its scenes' (Virilio 2008: 102) but, unlike Battlezone, as the gamer has no control over the movement of the vehicle, it is inertia which is substituted for mobility. This provides the vector for technological development. As with the UAV pilot or aeronaut the greater the speed the less the mobility: 'it is movement that governs the event (of war) and it is movement which produces the weaponry' (Virilio 2008: 112).

Operation Wolf, like Battlezone before with its present/absent logistical tallying uses the battlefield image so that it becomes impossible to imagine war without images. If, as discussed above, information is pure speed, then 
speed becomes war in its purest state, that is, pure war. The movement towards this zenith of speed, is a movement to a perpetual state of emergency of a war which is everywhere, but where the front is absent. In Operation Wolf when the damage to the gamer/warrior reaches a critical level, gauges flash and an alarm sounds, alerting the gamer/ warrior to the arena of danger but distancing any physical damage to the body of the gamer/warrior, projecting a psychological, ego-centric terror onto the individual; such is the manifestation of pure war a 'vectorial image of a combat without battle, but not without fear, that gives rise to an extermination that extends throughout the world' (Virilio 2008: 56). Operation Wolf adroitly realizes this. The final two levels are called 'Concentration Camp' and 'Airport' respectively, where the aim is to liberate hostages and then airlift them to safety. The pairing of two of the 20th century's most awesome technologies does not appear to be accidental and acts as a template for the world we now inhabit. It is not only the etymology which betrays the similarities, but the experience of those there which renders any differences between aviation and extermination nugatory. The tyranny of pure speed, is an intensification, a concentration: the arrival at the concentration camps of Oświęcim is, for train-passengers, quite literally, the terminal. Concurrently, the airport terminal concentrates speed of aviation and of processes. Airports, through the will-to-control of regulation, of prohibition, 'have the tragic character of the extermination camps' (Virilio 2008: 97) embodied in the use of pseudo-paramilitary uniformed personnel who are so ubiquitous that they pass into invisibility. Power is exercised with maximal immutability and minimal dissension and the more that mobility increases and is extended to greater and greater spaces, the more the demand for control intensifies. Pure speed is pure war is pure control: the endo-colonization of individuals, groups, races, entire societies renders every one of us a passenger, accelerating but inert, the speed of technocratic society an extended network of collapsed space and intensified time, generating users of inertia, exercised only through social control.

\section{| Endgame}

There is little wonder then that the increasing popularity of the videogame, linked to its dizzying clock speeds and verdant graphics has caught up, surpassed and now informs the state of emergency. Pure wars are inaugurated and performed every day and night between millions of people as the first person shooter series Call of Duty is played on Xbox Live and PlayStation Network. With proliferating digital spaces, there are also additional spaces for pure war to take place: gamers themselves are subjects of targeted advertising and national security surveillance as their online play and communication is monitored by security agencies from the US and UK. The monitoring of these communities is justified by GCHQ and NSA (in an ironic, if unintended deference to Virilio) through their protest that the enemy is everywhere, but cannot be seen. Being camouflaged and out-of-sight, much like a tank commander or a paramilitary illuminates the totality of pure war in videogames: agencies of the state are so afraid of their own tactics being used against them that the image of Operation Wolf moves from representation to reality. Gamers, G-men, and politicians and soldiers, informed by reams of metrics on Xbox dashboards become gamer/ warriors projected into digital space. Orbiting the battlefield, surrounded by a bubble of communication, distanced from lived space, separated from physical interaction, all are implicit in generating a 'concentration camp of speed [where] segregation and incarceration stem far more from the violence of displacement than from various police controls' (Virilio 2008: 57). This experience of the dromology of the space and time of the videogame can be acutely recalled in what is arguably the apogee of the series, Call of Duty 4's subtitle: Modern Warfare.

\section{Endnotes}

1. It is interesting to note that Mirage is the name given to the French Air Force's multirole fighter, itself deployed in a range of desert conflicts since its introduction in 1984. 


\section{Games}

\section{Battlezone(1980). Atari, Arcade}

- Call of Duty 4(2007) Infinity Ward, Microsoft Xbox 360

- $\quad$ Combat (1977) Atari, Atari VCS, 1977

- Duck Hunt (1984) Nintendo, Nintendo Entertainment System

- Gran Trak 10 (1974) Atari, Arcade,

- Gran Turismo (1997) Polyphony Digital, Sony PlayStation

- Monaco GP (1980) Sega/Gremlin Industries, Arcade

- Motor Mania (1982) John A. Fitzpatrick, Commodore 64

- $\quad$ Operation Wolf (1987) Taito, Arcade, 1987

- Outrun (1986) AM-2/Sega Arcade, 1986

- Parallax (1982) Sensible Software, Commodore 64
- Pole Position II (1983) Atari/Namco, Arcade

- R-Type (1987) Irem, Arcade

- $\quad$ Sprint 2(1976) Kee Games, Arcade

- TX-1 (1983) Tatsumi (Manufactured under license from Namco by Atari Inc),

Arcade

- WipEout HD (2008) Sony, PlayStation 3

- Zaxxon(1982) Sega, Arcade

\section{References}

Der Derian, James (2001) Virtuous War: Mapping the MilitaryIndustrial-Media-Entertainment-Network, Boulder: Westview Press

Schroter, Jens, (2014) 3D History, Theory and Aesthetics of the Transplane Image London: Bloomsbury

Stahl, Roger, (2010) Militainment Inc: War, Media and Popular Culture, New York: Routledge

Virilio, Paul. (1995) The Art of the Motor, Minnesota: University of Minnesota Press

Virilio, Paul. (1986) Speed and Politics, New York: Semiotext(e)
Virilio, Paul, (1997) Open Sky, London: Verso

Virilio, Paul, (2000a) Polar Inertia, London: Sage

Virilio, Paul, (2000b) The Information Bomb, London: Verso

Virilio, Paul, (2008) Negative Horizon, London: Continuum 
